\title{
Magnetic reconnection associated fluctuations in the deep magnetotail: ARTEMIS results
}

\author{
Z. Vörös \\ Institute of Astro- and Particle Physics, University of Innsbruck, Austria \\ Received: 30 September 2011 - Accepted: 11 November 2011 - Published: 24 November 2011
}

\begin{abstract}
On the basis of ARTEMIS two-probe mission magnetic reconnection (MR) outflow associated magnetic fluctuations and turbulence are analyzed on 19 February 2011. In the deep-tail, at distances between $X=45-$ $51 R_{\mathrm{E}}$, evidence for reconnection associated plasma sheet thinning was found, accompanied by heating of the plasma sheet. Correlated flow and field reversals and the large-scale Hall-effect signatures indicated the presence of the reconnection $X$-line. Within fast reconnection plasma outflows, magnetic fluctuations exhibit the same spectral scaling features and kinked spectra as magnetic fluctuations in the solar wind or in various parts of geospace. It was shown that the proton scale magnetic fluctuations are constrained by oblique firehose, proton cyclotron and mirror instability thresholds. For parallel plasma $\beta_{\|}>1$, where the thresholds converge, perpendicular magnetic fluctuations are enhanced. Magnetic compressibility decreases with the distance to the neutral sheet, however, near the instability thresholds it is comparable to the values obtained in the solar wind.
\end{abstract}

\section{Introduction}

Magnetic reconnection (MR) represents a multi-scale plasma process, in which the stored energy in the magnetic field is abruptly converted to plasma and particle energy, while the underlying system is usually embedded in a fluctuating and turbulent plasma environment. MR is associated with largescale (system- and fluid-scale) reorganizations of the magnetic field topology and localized kinetic (ion- and electronscale) processes, leading to particle energization and intense energy bursts affecting the system-scales (e.g. Zweibel and Yamada, 2009). MR is observed "in-situ" in the planetary

Correspondence to: Z. Vörös

(zoltan.voeroes@uibk.ac.at) magnetospheres and in the solar wind. It plays a crucial role in understanding the non-steady interaction of solar wind with the Earth's magnetosphere and in explaining the bursty response of magnetosphere-ionosphere system during magnetospheric storms and substorms.

In the Earth's magnetotail MR is preferentially initiated in the mid-tail $\left(X \sim-15\right.$ and $-30 R_{\mathrm{E}}$; where $R_{\mathrm{E}}$ is the Earth's radius). This region and the near-Earth magnetosphere are the most studied places in the tail, where MR represents a key element in energy accumulation and release during substorms. The multi-scale physics of MR was studied by recent multi-point Cluster and Themis missions, allowing to distinguish spatial and temporal variations and to examine the time sequence of the associated events. Deep-tail observations of the Geotail probe revealed an unsteady magnetotail at the distance of $X \sim-60 R_{\mathrm{E}}$ as well. It was found, that the tailward progression and retreat of MR associated activity is highly dynamic, turbulent, characterized by complex 3-D structures, multiple $X$-lines, appearance and dynamical evolution of acceleration centers (Angelopoulos et al., 1996). An Earthward moving $X$-line was also observed by the WIND spacecraft at $X \sim 60 R_{\mathrm{E}}$ (Farrell et al., 2003).

In laboratory, space and astrophysical plasmas the magnetic energy release is fast. For example, the MR associated electromagnetic energy can be released on a time scales of minutes in solar flares. The characteristic time scale of MR associated dynamic phenomena is also $1-3 \mathrm{~min}$ in the Earth's magnetotail (Sharma et al., 2008). Typically, bursty reconnection outflows last in a spacecraft frame only a few minutes in the mid-tail (Baumjohann et al., 1990). In collisionless space plasmas fast MR can occur due to different mechanisms, such as: slow shocks, anomalous resistivity, two-fluid (Hall) effects, turbulence, plasmoid formation, multiple reconnection, etc. Conceivably, various simultaneously occurring mechanisms, e.g. two-fluid effects combined with turbulence, can increase the reconnection rate, too. 

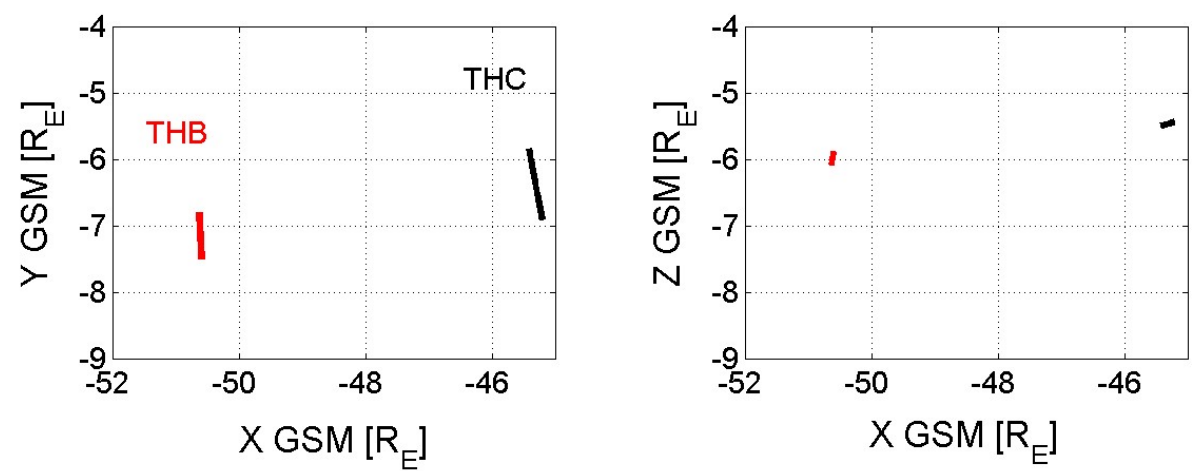

Fig. 1. Positions of the ARTEMIS probes in the GSM coordinate system.

Turbulence can be driven by MR itself or by external processes. Strong magnetic turbulence has already been observed within MR outflows in the mid-tail (Vörös et al., 2004). Mid-tail turbulence shows intermittent and anisotropy features (Vörös et al., 2003, 2008). The reconnection jets are accelerating ions, leading to ion-ion kink-like instability (Vörös et al., 2009). High-frequency turbulence observed occasionally within the ion diffusion region is consistent with fast mode whistler waves (Eastwood et al., 2009). Still, the observation of turbulence is rather difficult in the tail. A reliable statistical description of turbulent fluctuations requires long time series while the observations should reflect the properties of a steady turbulence. Frequent plasma sheet boundary crossings make the observations difficult. Multipoint turbulence observation techniques do not work when the spacecraft are in different physical regions separated by boundaries. Also, the magnetotail flows are mostly subAlfvenic, therefore single-point measurements techniques have strong limitations.

While moving tailward, MR associated plasmoids and reconnection jets can evolve towards more relaxed states (Slavin et al., 1995) and the outflows on the earthward side are not experiencing the strong dipole-like field. Therefore, the statistical analysis of turbulence at larger downtail distances can eventually rely more on longer measurements of steady physical processes. In this paper we investigate reconnection outflow associated turbulence using the data from two Artemis probes (Sibeck et al., 2011) in the deep-tail. First, we examine two-point measurements of a MR event and its magnetic field and plasma signatures, including the out-of-plane Hall field. Then the scaling properties of magnetic turbulence will be investigated jointly with gyroscale fluctuations constrained by pressure-anisotropy-driven instabilities.

\section{Observation of magnetic reconnection in the deep-tail}

\section{$2.1 \quad$ ARTEMIS data}

The "Acceleration, Reconnection, Turbulence and Electrodynamics of Moon's Interaction with the Sun" (ARTEMIS) NASA mission addresses the dynamics, scale size and energy balance of deep-tail particle acceleration and reconnection processes, solar wind and magnetotail turbulence, and the yet unknown kinetic properties of the lunar wake (Sibeck et al., 2011). The two ARTEMIS probes formed the part of the THEMIS five spacecraft fleet. After finishing the prime mission goals the THB and THC probes (also referred to as $\mathrm{P} 1$ and $\mathrm{P} 2$ ) were gradually placed into stable lunar orbits $\left(-60 R_{\mathrm{E}}\right.$ in the deep tail).

The MR event we analyze occurred on 19 February 2011, when the two probes were at distances $X \sim-45 R_{\mathrm{E}}$ and $X \sim-51 R_{\mathrm{E}}$ (Fig. 1) (the GSM coordinate system is used throughout this paper). The distances between the probes were $\Delta X=5.3 R_{\mathrm{E}}, \Delta Y=0.6 R_{\mathrm{E}}, \Delta Z=0.3 R_{\mathrm{E}}$, respectively. Since the average spatial scale of high-speed flows is $2-3 R_{\mathrm{E}}$ in the dawn-dusk direction and $1.5-2 R_{\mathrm{E}}$ in the north-south direction (Nakamura et al., 2004), there was a good chance to observe the reconnection outflows by both probes.

In this paper we use the data from the Flux-Gate magnetometer (FGM, Auster et al., 2008), the Search-Coil magnetometer (SCM, Roux et al.,2008), and the ion and electron Electrostatic Analyzer (ESA, McFadden et al., 2008). Magnetic data with time resolution of $1 / 4$ and $3 \mathrm{~s}$ (FGM) and $1 / 8 \mathrm{~s}$ (SCM) are used. The ESA instruments measure plasma over the energy range from a few $\mathrm{eV}$ up to $25 \mathrm{keV}$ for ions and up to $30 \mathrm{keV}$ for electrons.

\subsection{Overview of reconnection observations}

The reconnection event including the outflow jets occurred between 07:10 and 08:00 UT on 19 February 2011. The pseudo-AE index (not shown), calculated from the THEMIS magnetometer array (Russel et al., 2008) started to increase 


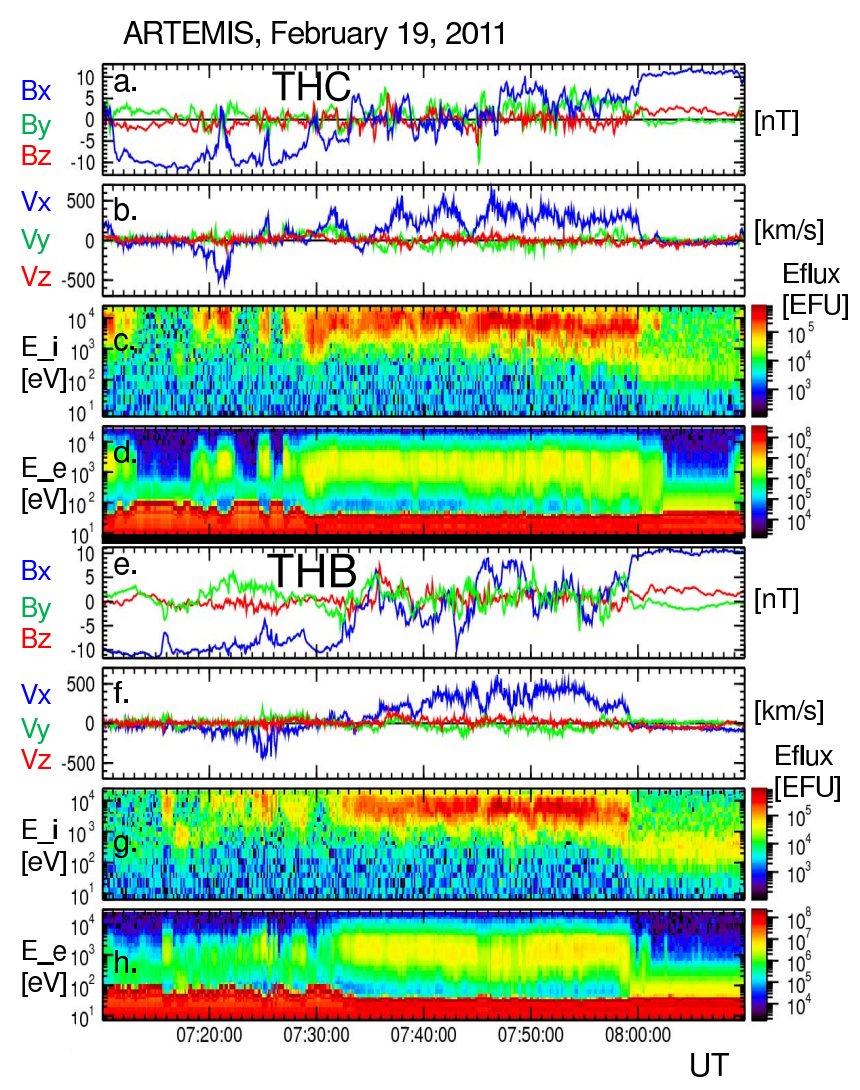

Fig. 2. ARTEMIS observations; (a-d) THC probe; (e-h) THB probe; (a) and (e) magnetic field GSM components; (b) and (f) plasma speed GSM components; $(\mathbf{c}, \mathbf{d})$ and $(\mathbf{g}, \mathbf{h})$ ion and electron energy flux spectrograms; EFU stays for Energy Flux Unit in $\left(\mathrm{eV} / \mathrm{cm}^{-2}\right.$.s.sr.eV).

gradually already at 04:00 UT reaching its maximum values of $\mathrm{AE} \sim 400 \mathrm{nT}$ between 08:00 and 09:00 UT. The plasma sheet was active before 07:10 UT (not shown), but in that time period, both probes were predominantly close to the plasma sheet boundary layer. Figure 2 shows the ARTEMIS THC (a-d) and THB (e-h) observations. For both spacecraft, the spin-resolution magnetic field components (Fig. 2a and e), the bulk speed components (Fig. 2b and f) ion (Fig. 2c and $g$ ) and electron (Fig 2d and h) energy flux spectrograms from the ESA instruments are shown.

The spacecraft are gradually crossing the plasma sheet, observing initially $B_{X} \sim-10 \mathrm{nT}$ in the Southern Hemisphere then $B_{X} \sim+10 \mathrm{nT}$ in the Northern Hemisphere. Minimum variance analysis calculations applied for the crossings of the neutral sheet $\left(B_{X}=0 \mathrm{nT}\right)$ showed that the neutral sheet normal was close to GSM $Z$ direction. This shows that the GSM coordinate system is appropriate for the analysis of this event.

Between 07:20 and 08:00 UT both spacecraft observe high-speed flow reversals. Tailward flows reaching $V_{X} \sim$ $-500 \mathrm{~km} \mathrm{~s}^{-1}$ are observed by both spacecraft. A high-speed tailward flow appears at the position of THC first (closer to the Earth), the reconnection $X$-line is most likely moving from THC towards THB, tailward (Fig. 2b and f). However, precise two-point timing analysis for these flows is not possible because of the relative motion of the plasma sheet and the probes. Before $\sim 07: 30 \mathrm{UT}$, the $B_{X}$ components show that the probes are in the central plasma sheet only for a short time at about 07:01 UT (THC) and at about 07:05 (THB). During this interval the ion and electron energy flux spectrograms show only intermittent fluxes of higher energy particles (Fig. 2c, d, g and h). All that indicate that high-speed tailward flows can exist earlier or later than the observed flows, the probes are just intermittently inside/outside of the flow channel.

During the extended period of Eathward flows, reaching $\sim 500 \mathrm{~km} \mathrm{~s}^{-1}$ maxima roughly between 07:30 and 08:00 UT, the probes are inside the plasma sheet and the flow channels. The probes gradually cross the neutral sheet, while energetic ions and electrons are abundantly observed, up to the limiting energies of the ESA instrument. Energetic particles and Earthward flows are observed by THC at $\sim 07: 30$ UT first, but the large absolute value of $B_{X}$ at the same time indicates that THB is outside of the plasma sheet. For the same reason the Earthward flows observed by THB end earlier than the THC flows, at 07:29 UT.

Plasma $\beta$ time series in Fig. 3g support the same picture. Between 07:34 and 07:58 UT, except for short deviations at 07:36 and 07:46 UT, both THB (red line) and THC (black line) observe a fluctuating plasma $\beta \geq 1$. The plasma $\beta$ is often used as a proxy of the distance of a spacecraft from the neutral sheet. Statistical results show that $\beta \geq 0.3$ values indicate a transition from the plasma sheet boundary layer through outer plasma sheet $(\beta \sim 0.3)$ towards the central plasma sheet $(\beta \sim 3)$ and the neutral sheet $(\beta \sim 30)$ (Baumjohann et al., 1989; Snekvik et al., 2007). In this sense, the horizontal dashed line $(\beta=1)$ in Fig. $3 g$ roughly indicates the plasma sheet encounter by the probes (Baumjohann, 1993).

Figure 3 also compares the smoothed total magnetic field $B_{\mathrm{S}}$ (Fig. 3a), the smoothed $B_{X S}$ (Fig. 3b), $B_{Z}$ (Fig. 3c), $V_{X}$ (Fig. 3d), the ion density $N$ (Fig. 3e) and the ion temperature $T_{\mathrm{i}}$ with plasma $\beta$ (Fig. $3 \mathrm{~g}$ ). Despite the fluctuations visible in the smoothed $B_{\mathrm{S}}$ and $B_{X \mathrm{~S}}$, THB (red) and THC (black) observe similar large-scale magnetic profiles indicating the simultaneous crossings of the plasma sheet. Before 07:20 and after 08:00 UT the $B_{X S}$ values are similar, larger differences in $B_{X S}$ are associated with increased plasma $\beta$. The increased differences between two-point values of $B_{X \mathrm{~S}}$ indicate a steepening of the gradient in $Z$ direction due to a thinning of the plasma sheet and increasing current density. Since it occurred in conjunction with the flow reversal, we can speculate that the formation of the thin current sheet facilitated collisionless MR. The density and ion temperature measurements (Fig. 3e and g) show that, before 07:30 UT, the plasma was tenuous the ion temperature intermittently increased during the occasional excursions to the plasma sheet. 


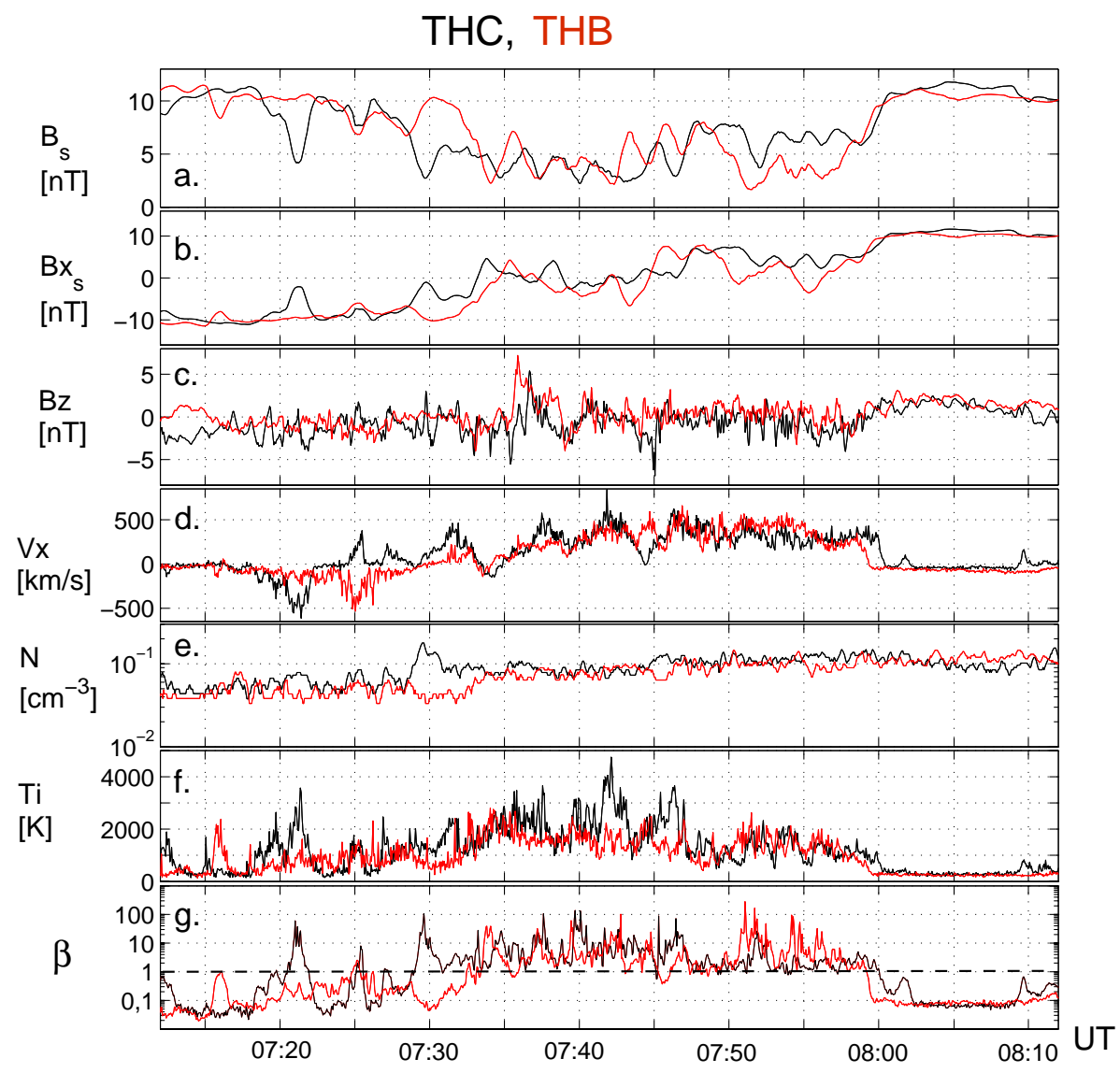

Fig. 3. Comparison of THC (black lines) and THB (red lines) probe measurements; (a) smoothed magnetic field magnitude; (b) smoothed $B_{X}$ magnetic field component; (c) $B_{Z}$ magnetic field component; (d) $V_{X}$ component of the bulk speed; (e) ion density; (f) ion temperature; (g) plasma $\beta$.

The same intermittent behavior is visible in the ion and electron energy flux spectrograms in Fig. $2 \mathrm{c}, \mathrm{d}$, g, and h before $07: 30$. The extended period of tailward flow after $07: 30$ is associated with a dense and hot plasma sheet.

For the identification of MR $X$-line the flow reversal is a necessary but not sufficient condition. Large-scale flow vortices associated with bursty flows in the magnetotail can lead to a flow reversal without any $X$-line encounter (Birn et al., 2004). A statistical analysis of plasma flow data between 9 and $19 R_{\mathrm{E}}$ has shown that MR is accompanied by fast Earthward flows with positive $B_{Z}$ (magnetic field dipolarization) and fast tailward flows with negative $B_{Z}$ (Baumjohann et al., 1990). Figure 3c shows that a significant dipolarization observed by both THB and THC is associated with the Earthward flow (Fig. 3d ) between 07:35 and 07:40. The other field signatures are less significant because of the fluctuating magnetic field.

To further investigate the large-scale signatures of MR scatter-plots of $V_{X}$ versus $B_{Z}$ and $V_{X}$ versus $B_{X}$ for $B_{Y}>0$ and $B_{Y}<0$ are presented in Fig. 4. In order to consider plasma sheet intervals, data values associated with plasma $\beta \geq 1$ are plotted only. Figure $4 \mathrm{a}$ and $\mathrm{b}$ shows these data for THB and Fig. 4c and d for THC, respectively. Figure 4a and $\mathrm{c}$ shows that both probes observe tailward flows correlated with southward magnetic field $\left(-V_{X}\right.$ vs. $\left.-B_{Z}\right)$. There is a large population of points showing the correlation between Earthward flows and northward magnetic field $\left(+V_{X}\right.$ vs. $+B_{Z}$, mainly THB), but there is also a significant population of data points (mainly from THC) in the $+V_{X}$ vs. $-B_{Z}$ quadrant. Actually, dipolarization is expected to occur predominantly at the leading edge of high-speed Earthward flows, the trailing edge of flow bubbles can be associated with mixed $\pm B_{Z}$ fluctuations. Figure $4 \mathrm{~b}$ and d shows the outof-plane magnetic field $B_{Y}$ associated with the Hall current system near MR in $V_{X}$ vs. $B_{X}$ scatter-plots. Within Earthward flows, the Hall current system leads to the out of plane $B_{Y}>0$ magnetic field in Northern Hemisphere $\left(B_{X}>0\right)$ and $B_{Y}<0$ in the Southern Hemisphere $\left(B_{X}<0\right)$. The correlations are opposite within tailward flows (e.g. Runov et al., 2003). It means that, in Fig. $4 \mathrm{~b}$ and d, the top-left and the bottom-right quadrants should contain only $B_{Y}<0$ values (red points) and the top-right and the bottom-left quadrants 

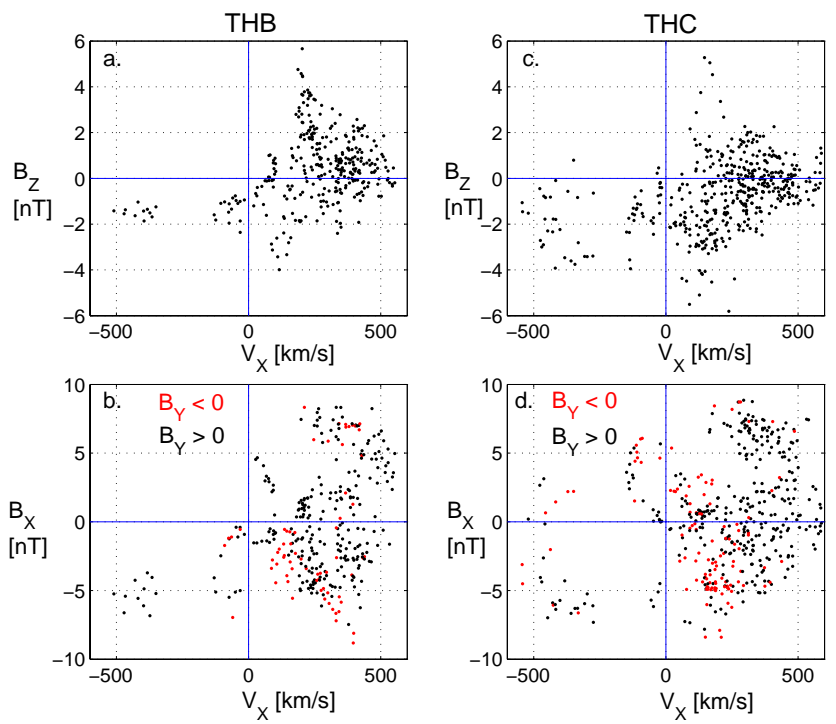

Fig. 4. Field-velocity scatter-plots, (a, b) THB probe; (c, d) THC probe; (a) and (c) $V_{X}$ versus $B_{Z}$; (b) and (d) $V_{X}$ versus $B_{X} ; B_{Y}<0$ - red points; $B_{Y}>0$ - black points.

only $B_{Y}>0$ values (black points). This is the so-called quadrupolar magnetic structure associated with the Hall currents. For both THB and THC probes mainly the bottomleft quadrant is contaminated by $B_{Y}>0$ black points, otherwise the Hall out-of-plane magnetic signatures are recognizable. Eastwood et al. (2010) presented similar scatter-plots on the basis of a wider statistical study using 33 correlated field and flow reversals from Cluster in the mid-tail. In their larger data set the quadrupolar out-of-plane Hall magnetic field is more apparent than here, however, their scatter-plots are also contaminated by $B_{Y}$ data of improper sign. Also, in our case the amplitude of magnetic fluctuations exhibiting 1-5 min periodicity is rather significant during the Earthward flow (Fig. 3a and b), which can explain the deviations from the expected quadrupolar structure.

\section{Earthward flow associated fluctuations/turbulence}

Since any statistical analysis of fluctuations requires long enough time series, the extended interval of Earthward flow seems to be a good choice for studying the scaling properties of fluctuations. The $X$ separation of THB and THC probes is large, therefore we avoid joint two-point statistics and use single-point considerations instead. In the super-Alfvenic solar wind time series obtained from a single spacecraft are used for mapping the spatial structure of turbulence along the flow. Over the magnetohydrodynamic scales Taylor's hypothesis ensures that the spatial fluctuations in an Alfvenic turbulence pass over the spacecraft faster than they typically fluctuate in time. In the plasma sheet the Taylor's hypothesis can be used during intervals of fast plasma flows (Vörös et al., 2006).

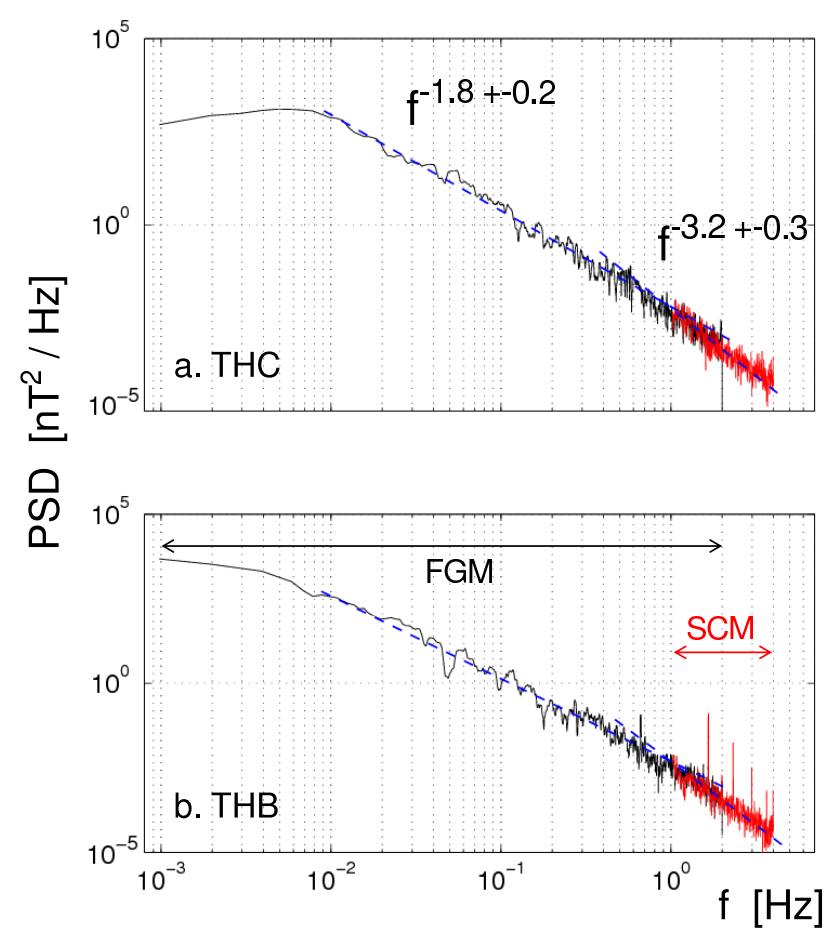

Fig. 5. Power spectral density (PSD) plots; (a) THC probe; (b) THB probe; FGM and SCM magnetic instruments data are used.

Figure 5a and $\mathrm{b}$ shows the power spectral density (PSD) plots of the magnetic field for THC and THB, respectively. The spectra were obtained using the combined FGM (black line) and SCM (red line) magnetometer $B_{X}$ data during the Earthward flow period in Fig. 3d. The inclusion of the tailward flows does not change the spectra. The intriguing feature of the spectra is that low-frequency fluctuations exhibit Kolmogorov-like scaling with spectral index $1.8 \pm 0.2$, while the power is decreasing more rapidly over high-frequencies and the spectral index is $3.2 \pm 0.3$. There is no difference between THC and THB spectra. Similar kinked spectra were observed in the solar wind (Leamon et al., 1998; Alexandrova et al., 2009) and in the geospace environment (see the review by Zimbardo et al., 2010). In the mid-tail similar kinked spectra were obtained only recently (Vörös et al., 2007). It is an accepted view that the low-frequency scaling appears as a consequence of nonlinear turbulent interactions over magnetohydrodynamic scales, the high-frequency scaling is the result of interactions in kinetic turbulence. The latter interactions are less understood, but in general, the question is how the cascading energy from the fluid scales reaches the ion and electron scales and what is the nature of kinetic scale cascade and energy dissipation. The different scenarios include a kinetic cascade built up by perpendicularly propagating kinetic Alfven waves (Howes et al., 2008), weakly damped magnetosonic/whistler modes (Stawicki et al., 2001), etc. Since it is not fully known what kind of 
spatial fluctuations exist in kinetic turbulence, the application of Taylor's hypothesis over kinetic scales is questionable. Therefore, the kinked power spectra obtained from one-point measurements should be interpreted cautiously. Though multi-point Cluster observations allowed to reproduce the spectral scalings in wavevector space without assuming the validity of Taylor hypothesis (Sahraoui et al., 2010), the whole kinetic range was not covered due to the large spatial separation of the spacecraft. The multi-point observations of turbulence, however, confirmed that the observed $k$-spectra were strongly anisotropic favoring the occurrence of small-scale perpendicular to magnetic field fluctuations. It was also conjectured that part of the energy of the magnetohydrodinamic turbulent cascade is dissipated at proton scales via Landau damping and the remaining part of the energy is cascading down towards the electron scales (Sahraoui et al., 2010).

In the magnetotail, scale-dependent anisotropy was also observed on the basis of single-point measurements, using the normalized strength of magnetic fluctuations as a proxy for $k$-vector anisotropy (Vörös et al., 2008). Based on the turbulence model of Oughton et al. (1998) the anisotropy of fluctuations can be estimated through

$\cos ^{2} \Theta=m\left(\frac{\delta b}{B_{0}+\delta b}\right)^{2}+c$

where $B_{0}$ is the local mean magnetic field, $m$ and $c$ are constants (Oughton et al., 1998) and the anisotropy angle $\Theta=0^{\circ}$ is associated with parallel while $\Theta=90^{\circ}$ with purely perpendicular to magnetic field fluctuations. During our plasma sheet crossing intervals the anisotropy angle associated with proton scale magnetic fluctuations varied between $\Theta=65$ $85^{\circ}$, indicating the quasi-perpendicular nature of underlying fluctuations.

Recently, it was found that, in the solar wind, the magnetic spectral scaling near or below the proton scales is influenced by pressure anisotropy driven instabilities (Bale et al., 2009). Specifically, it was discovered that gyroscale magnetic fluctuations in the solar wind are enhanced along the temperature anisotropy thresholds corresponding to mirror, proton oblique firehose, and ion cyclotron instabilities (Hellinger et al., 2006; Bale et al., 2009). The proton temperature anisotropy $T_{\perp} / T_{\|}$depends on proton parallel beta $\beta_{\|}$(defined through parallel proton temperature) and the instability thresholds were fitted for a marginal stability condition (Hellinger et al., 2006;):

$\frac{T_{\perp}}{T_{\|}}=1+\frac{a}{\left(\beta_{\|}-\beta_{0}\right)^{b}}$

where $\left(a, b, \beta_{0}\right)=(0.43,0.42,-0.0004)$ for proton cyclotron, $\left(a, b, \beta_{0}\right)=(0.77,0.76,-0.016)$ for mirror and $\left(a, b, \beta_{0}\right)=(-1.4,1,-0.11)$ for oblique firehose instability.

In Fig. 6 we depicted the distribution of ion temperature anisotropy $T_{\perp} / T_{\|}$from the ARTEMIS ESA instrument with respect to the parallel $\beta_{\|}$during the intervals of high-speed

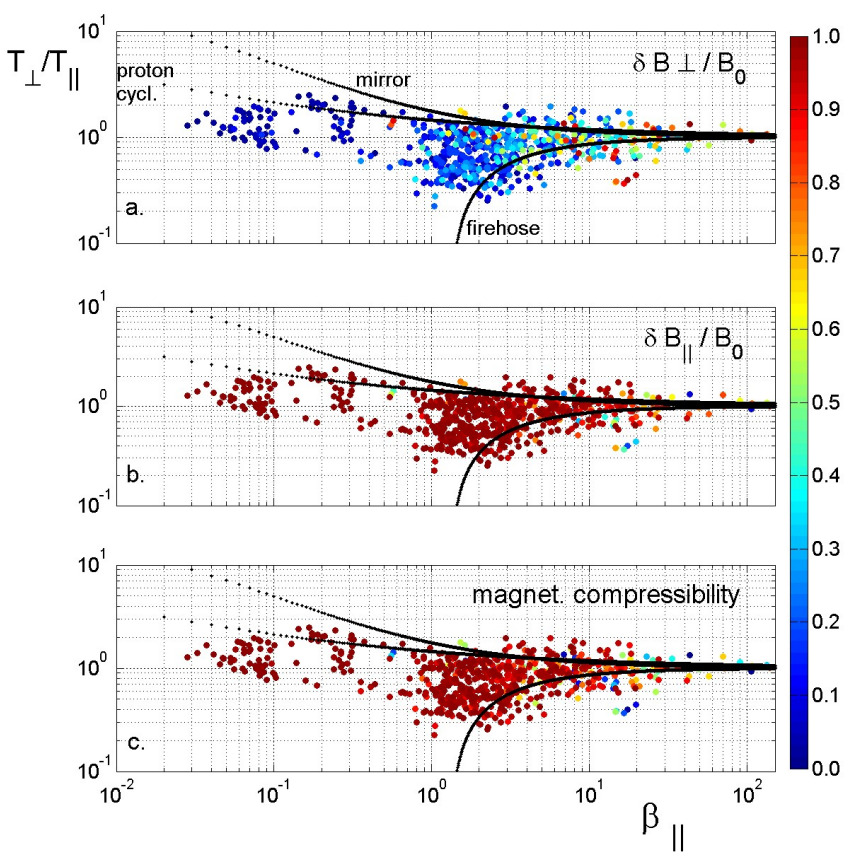

Fig. 6. Proton temperature anisotropy versus parallel plasma $\beta_{\|}$. The color code corresponds to the strength of (a) normalized perpendicular magnetic fluctuations; (b) normalized parallel magnetic fluctuations; (c) magnetic compressibility. The dotted lines correspond to proton cyclotron, mirror and oblique firehose instability thresholds.

flows. We note that ARTEMIS is unable to distinguish protons and ions, however, we can suppose that the observed ions are mostly protons. The color code in Fig. 6a corresponds to the normalized rms magnetic fluctuations perpendicular to the mean magnetic field $\delta B_{\perp} / B_{0}$. The mean field $B_{0}$ is computed at the time-scale of $10 \mathrm{~s}$ from the magnetic field vector available with time resolution of $1 / 4 \mathrm{~s}$ from the FGM instrument. The color code in Fig. 6b corresponds to the normalized rms parallel magnetic fluctuations $\delta B_{\|} / B_{0}$. Lastly, Fig. 6c shows magnetic compressibility defined through $\delta B_{\|}^{2} /\left(\delta B_{\|}^{2}+\delta B_{\perp}^{2}\right)$. There was no significant difference between the data from THB and THC, therefore, the three subplots in Fig. 6 contain the data from both probes. The dotted lines indicates the proton cyclotron and mirror instability thresholds for $T_{\perp} / T_{\|}>1$ and the oblique firehose instability threshold for $T_{\perp} / T_{\|}<1$. The data distributions in Fig. 6 are constrained by the indicated temperature anisotropy instability thresholds. This means that a plasma, exhibiting anisotropies higher or lower than the indicated thresholds, would be unstable and drive fluctuations via proton cyclotron, mirror and oblique firehose instabilities, relaxing towards stable states with temperature anistropies closer to one.

The plasma $\beta_{\|}$values can be considered as a rough proxy of the distance from the neutral sheet, as explained above for $\beta$. In this sense, the fluctuations near the lobe, the plasma sheet boundary layer, and the outer plasma sheet $\left(\beta_{\|} \leq 1\right)$ 
seem to be constrained by the proton cyclotron instability threshold rather than by the mirror instability threshold for $T_{\perp} / T_{\|}>1$. In the plasma sheet or close to the neutral sheet, where $\left(\beta_{\|} \gg 1\right)$, these two instability thresholds are indistinguishable, but still represent constraints for the data distribution. For $T_{\perp} / T_{\|}<1$, the data are mainly restricted to the plasma sheet as for the majority of points $\beta_{\|} \geq 1$ and the constraint is represented by the oblique firehose instability threshold. Few points are deviating significantly from the firehose instability threshold near $\beta_{\|} \sim 20$, these data can be associated with the processes in the current sheet. This has to be investigated using a larger set of similar events. The contribution of perpendicular magnetic fluctuations to the mean field is negligible outside of the plasma sheet $\left(\beta_{\|} \leq 1\right)$ (Fig. 6a), parallel, highly compressional magnetic fluctuations dominate (Fig. $6 \mathrm{~b}$ and c). Within the plasma sheet and closer to the neutral sheet $\left(\beta_{\|} \geq 1\right)$, magnetic compressibility decreases and the perpendicular magnetic fluctuations are getting stronger. At high values of $\beta_{\|}$, where the thresholds converge, perpendicular magnetic fluctuations are enhanced. These plots are similar to those presented by Bale et al. (2009) for the case of proton temperature anisotropies in the solar wind. Magnetic compressibility in the solar wind is predominantly enhanced along the mirror instability threshold at high plasma $\beta$. Nevertheless, as magnetic compressibility in the plasma sheet (Fig. 6c) decreases (for $\beta_{\|}>1$ ), it reaches values between 0.2 and 0.6 . These are very similar to the enhanced values of magnetic compressibility along mirror and oblique firehose instability thresholds in the solar wind (Bale et al., 2009). However, contrarily to the solar wind, away from the instability thresholds magnetic compressibility in the plasma sheet is large, it reaches values between 0.7 and 1 .

\section{Conclusions}

In this paper MR associated fluctuations and turbulence were analyzed. The overview of ARTEMIS two-probe magnetic field, particle and plasma measurements was presented. Evidence was found for plasma sheet thinning during MR, correlated flow and field reversals, including the Hall-effect. During the fast flows, magnetic fluctuations exhibit the same scaling features and kinked PSD as magnetic fluctuation spectra in the solar wind or in various parts of the geospace. The kinked spectra were not routinely observed in the midtail plasma sheet (Vörös et al., 2007).

It has been found by Bale et al. (2009) that magnetic fluctuation power near proton scales is constrained by temperature anisotropy instability thresholds. It indicates, that the energy transfer via nonlinear cascades or the transition from magnetohydrodinamic to kinetic scales is influenced by fluctuations driven by temperature anisotropy instabilities as well. Compressibility is mainly enhanced along the mirror instability threshold in the solar wind.
This study shows that, reconnection associated magnetic fluctuations within fast plasma flows in the deep tail exhibit kinked spectra and the scalings can presumably be associated with plasma turbulence over magnetohydrodynamic and kinetic scales. Similarly to the solar wind case, the proton scale fluctuations, where the cascading energy from the magnetohydrodynamic scales reaches the kinetic scales, is influenced by proton temperature anisotropy instabilities.

Near the plasma sheet boundary layer the proton scale fluctuations are constrained by the proton-cyclotron instability threshold. Within the plasma sheet $(\beta>1)$, perpendicular magnetic fluctuations are enhanced and the fluctuations become less compressional. However, magnetic compressibility along the instability thresholds in the plasma sheet reaches the same values as magnetic compressibility in the solar wind. The proton scale fluctuations are constrained by oblique firehose instability for $T_{\perp} / T_{\|}<1$, while the constraints introduced by proton cyclotron and mirror instability thresholds are the same for $T_{\perp} / T_{\|}>1$.

Compressional wave power dominates over the large magnetohydrodynamic scales in the plasma sheet. Fast plasma flows or/and substorm activity increase the spectral power of compressional waves (Volwerk et al., 2004). However, the compressional magnetic wave power and its dominance over non-compressional waves decreases towards higher frequencies. Our results show that within fast flows the level of perpendicular fluctuations increases when the probes are closer to the neutral sheet. Regarding the proton firehose instability, hybrid simulations of the solar wind $\beta \sim 1$ anisotropic plasma have shown that the oblique firehose strongly scatters the protons, therefore important portion of the largescale wave energy is dissipated to protons (Hellinger and Trávníček, 2008). This mechanism has to be taken into account in the magnetotail plasma sheet as well.

Acknowledgements. We thank A. Runov and M. Volwerk for helpful discussions. We acknowledge NASA contract NAS502099 and V. Angelopoulos for use of data from the THEMIS Mission. Specifically, C. W. Carlson and J. P. McFadden for use of ESA data, K. H. Glassmeier, U. Auster, and W. Baumjohann for use of FGM data, A. Roux and O. LeContel for use of SCM data. This work was made with the support of the ISSI team 185 "Dispersive cascade and dissipation in collisionless space plasma turbulence: ubservations and simulations". The work was also supported by the Austrian Wissenschaftsfonds FWF under Grant No. Y398.

Edited by: G. Lapenta

Reviewed by: G. Facskó and another anonymous referee 


\section{References}

Alexandrova, O., Saur, J., Lacombe, C., Mangeney, A., Mitchell, J., Schwartz, S. J., and Robert, P.: Universality of solar wind turbulence spectrum from MHD to electron scales, Phys. Rev. Lett., 103, 165003, doi:10.1103/PhysRevLett.103.165003, 2009.

Angelopoulos, V., Mitchell, D. G., McEntire, R. W., Williams, D. J., Lui, A. T. Y., Krimigis, S. M., Decker, R. B., Christon, S. P., Kokubun, S., Yamamoto, T., Saito, Y., Mukai, T., Mozer, F. S., Tsuruda, K., Reeves, G.D., Hughes, W. J., Friis-Christensen, E., and Troshichev, O.: Tailward progression of magnetotail acceleration centers: relationship to substorm current wadge, J. Geophys. Res., 101, 24599-24619, 1996.

Auster, H. U., Glassmeier, K. H., Magnes, W., Aydogar, O., Baumjohann, W., Constantinescu, D., Fischer, D., Fornacon, K. H., Georgescu, E., Harvey, P., Hillenmaier, O., Kroth, R., Ludlam, M., Narita, Y., Nakamura, R., Okrafka, K., Plaschke, F., Richter, I., Schwarzl., H., Stoll, B., Valavanoglou A., and Wiedemann, M.: The THEMIS fluxgate magnetometer, Space Sci.Rev., 141, 235-264, 2008.

Bale, S. D., Kasper, J. C., Howes, G. G., Quataert, E., Salem, C., and Sundkvist, D.: Magnetic fluctuation power near proton temperature instability thresholds in the solar wind, Phys. Rev. Lett., 103, 211101, doi: 10.1103/PhysRevLett.103.211101, 2009.

Baumjohann, W.: The near-Earth plasma sheet: an AMPTE-IRM perspective, Space Sci. Rev., 64, 141-163, 1993.

Baumjohann, W., Paschmann, G., and Cattell, C. A.: Average Plasma Properties in the Central Plasma Sheet, J. Geophys. Res., 94, 6597-6606, 1989.

Baumjohann, W., Paschmann, G., and Luhr, H.: Characteristics of high-speed ion flows in the plasma sheet, J. Geophys. Res., 95, 3801-3809, 1990.

Birn, J., Raeder, J., Wang, Y. L., Wolf, R. A., and Hesse, M.: On the propagation of bubbles in the geomagnetic tail, Ann. Geophys., 22, 1773-1786, doi:10.5194/angeo-22-1773-2004, 2004.

Eastwood, J. P., Phan, T. D., Bale, S. D., and Tjulin, A.: Observations of turbulence generated by magnetic reconnection, Phys. Rev. Lett., 102, 035001, doi:10.1103/PhysRevLett.102.035001, 2009.

Eastwood, J. P., Phan, T. D., Øieroset, M., and Shay, M. A.: Average properties of the magnetic reconnection ion diffusion region in the Earth's magnetotail: The 2001-2005 Cluster observations and comparison with simulations, J. Geophys. Res., 115, A08215, doi:10.1029/2009JA014962, 2010.

Farrell, W. M., Desch, M. D., Ogilvie, K. W., Kaiser, M. L., and Goetz, K.: The role of upper hybrid waves in magnetic reconnection, Geophys. Res. Lett., 30, 2259, doi:10.1029/2003GL017549, 2003.

Hellinger, P. and Trávníček, P.: Oblique proton fire hose instability in the expanding solar wind: Hybrid simulations, J. Geophys. Res., 113, A10109, doi:10.1029/2008JA013416, 2008.

Hellinger, P., Trávníček, P., Kasper, J. C., and Lazarus, A. J.: Solar wind proton temperature anisotropy: Linear theory and WIND/SWE observations, Geophys. Res. Lett., 33, L09101, doi:10.1029/2006GL025925, 2006.

Howes, G. G., Cowley, S. C., Dorland, W., Hammett, G. W., Quataert, E., and Schekochihin, A. A.: A model of turbulence in magnetized plasmas: Implications for the dissipation range in the solar wind, J. Geophys. Res., 113, A05103, doi:10.1029/2007JA012665, 2008.
McFadden, J. P., Carlson, C. W., Larson, D., Angelopoulos, V., Ludlam, M., Abiad, R., Elliott, B., Turin, P., and Marckwordt, M.: The THEMIS ESA plasma instrument and in-flight calibration, Space Sci. Rev, 141, 277-302, doi:10.1007/s11214-008-9440-2, 2008.

Leamon, R. J., Smith, C. W., Ness, N. F., and Wong, H. K.: Dissipation range dynamics: Kinetic Alfven waves and the importance of electron beta, J. Geophys. Res., 104, 22331-22344, 1999.

Nakamura, R., Baumjohann, W., Mouikis, C., Kistler, L. M., Runov, A., Volwerk, M., Asano, Y., Vörös, Z., Zhang, T. L., Klecker, B., Rème, H., and Balogh, A.: Spatial scale of high-speed flows in the plasma sheet observed by Cluster, Geophys. Res. Lett., 31, L09804, doi:10.1029/2004GL019558, 2004.

Oughton, S., Matthaeus, W. H., and Ghosh, S.: Scaling of spectral anisotropy with magnetic field strength in decaying magnetohydrodynamic turbulence, Phys. Plasmas, 5, 4235-4242, 1998.

Roux, A., Le Contel, O., Coillot, C., Bouabdellah, A., de la Porte, B., Alison, D., Ruocco, S., and Vassal, M. C.: The search coil magnetometer for THEMIS, Space Sci. Rev., 141, 265-275, doi:10.1007/s11214-008-9371-y, 2008.

Russell, C. T., Chi, P. J., Dearborn, D. J., Ge, Y. S., Kuo-Tiong, B., Means, J. D., Pierce, D. R., Rowe, K. M., and Snare, R. C.: Themis ground-based magnetometers, Space Sci. Rev., 141, 389-412, doi:10.1007/s11214-008-9337-0, 2008.

Sahraoui, F., Goldstein, M. L., Belmont, G., Canu, P., and, Rezeau, L.: Three dimensional anisotropic k-spectraof turbulence at subproton scales in the solar wind, Phys. Rev. Lett., 105, 131101, doi:10.1103/PhysRevLett.105.131101, 2010.

Sharma, A. S., Nakamura, R., Runov, A., Grigorenko, E. E., Hasegawa, H., Hoshino, M., Louarn, P., Owen, C. J., Petrukovich, A., Sauvaud, J.-A., Semenov, V. S., Sergeev, V. A., Slavin, J. A., Sonnerup, B. U. Ö., Zelenyi, L. M., Fruit, G., Haaland, S., Malova, H., and Snekvik, K.: Transient and localized processes in the magnetotail: a review, Ann. Geophys., 26, 9551006, doi:10.5194/angeo-26-955-2008, 2008.

Sibeck, D. G., Angelopoulos, V., Brain, D. A., Delory, G. T., Eastwood, J. P., Farrell, W. M., Grimm, R. E., Halekas, J. S., Hasegawa, H., and Hellinger, P., Khurana, K. K., Lillis, R. J., Øieroset, M., Phan, T.-D., Raeder, J., Russel, C. T., Schriver, D., Slavin J. A., Travnicek, P. M., and Weygand J. M.: ARTEMIS science objectives, Space Sci. Rev., doi:10.1007/s11214-0119777-9, first published online, 2011.

Slavin, J. A., Owen, C. J., Kuznetsova, M. M., and Hesse, M.: ISEE 3 observations of plasmoids with flux rope magnetic topologies, Geophys. Res. Lett., 22, 2061-2064, 1995.

Snekvik, K., Haaland, S., Østgaard, N., Hasegawa, H., Nakamura, R., Takada, T., Juusola, L., Amm, O., Pitout, F., Rème, H., Klecker, B., and Lucek, E. A.: Cluster observations of a field aligned current at the dawn flank of a bursty bulk flow, Ann. Geophys., 25, 1405-1415, doi:10.5194/angeo-25-1405-2007, 2007.

Stawicki, O., Gary, S. P., and Li, H.: Solar wind magnetic fluctuation spectra: Dispersion versus damping, J. Geophys. Res., 106, 8273-8281, doi:10.1029/2000JA000446, 2001.

Volwerk, M., Baumjohann, W., Glassmeier, K. H., Nakamura, R., Zhang, T. L., Runov, A., Vörös, Z., Klecker, B., Treumann, R. A., Bogdanova, Y., Eichelberger, H.-U., Balogh, A., and Rème, H.: Compressional waves in the Earth's neutral sheet, Ann. Geophys., 22, 303-315, doi:10.5194/angeo-22-303-2004, 2004.

Vörös, Z., Baumjohann, W., Nakamura, R., Runov, A., Zhang, T. 
L., Volwerk, M., Eichelberger, H. U., Balogh, A., Horbury, T. S., Glaßmeier, K.-H., Klecker, B., and Rème, H.: Multi-scale magnetic field intermittence in the plasma sheet, Ann. Geophys., 21, 1955-1964, doi:10.5194/angeo-21-1955-2003, 2003.

Vörös, Z., Baumjohann, W., Nakamura, R., Volwerk, M., Runov, A., Zhang, T. L., Eichelberger, H. U., Treumann, R., Georgescu, E., Balogh, A., Klecker, B., and Reme, H.: Magnetic turbulence in the plasma sheet, J. Geophys. Res., 109, A11215, doi:10.1029/2004JA010404, 2004.

Vörös, Z., Baumjohann, W., Nakamura, R., Volwerk, M., and Runov, A.: Bursty bulk flow driven turbulence in the Earths plasma sheet, Space Sci. Rev., 122, 301, doi:10.1007/s11214006-6987-7, 2006.

Vörös, Z., Baumjohann, W., Nakamura, R., Runov, A., Volwerk, M., Asano, Y., Jankovivccová, D., Lucek, E. A., and Rème, H.: Spectral scaling in the turbulent Earth's plasma sheet revisited, Nonlin. Processes Geophys., 14, 535-541, doi:10.5194/npg-14535-2007, 2007.
Vörös, Z., Nakamura, R., Sergeev, V., Baumjohann, W., Runov, A., Zhang, T.L., Volwerk, M., Takada, T., Jankovicova, D., Lucek, E., and Reme, H.: Study of reconnection-associated multiscale fluctuations with Cluster and Double Star, J. Geophys. Res., 113, A07S29, doi:10.1029/2007JA012688, 2008.

Vörös, Z., Leubner, M. P., Runov, A., Angelopoulos, V., and Baumjohann, W.: Evolution of kinklike fluctuations associated with ion pickup within reconnection outflows in the Earth's magnetotail, Phys. Plamsas, 16, 20701, doi:10.1063/1.3271410, 2009.

Zimbardo, G., Greco, A., Sorriso-Valvo, L., Perri, S., Vörös, Z., Aburjania, G., Chargazia, K., and Alexandrova, O.: Magnetic turbulence in the geospace environment, Space Sci. Rev., 156, 89-134, 2010.

Zweibel, E. G. and Yamada, M.: Magnetic reconnection in astrophysical and laboratory plasmas, Annu. Rev. Astron. Astrophys., 47, 291-332, 2009. 